\title{
Pertussis toxin inhibits neutrophil recruitment to delay antibody-mediated clearance of Bordetella pertussis
}

\author{
Girish S. Kirimanjeswara, ${ }^{1}$ Luis M. Agosto, ${ }^{1}$ Mary J. Kennett, ${ }^{1}$ Ottar N. Bjornstad, ${ }^{2}$ and Eric T. Harvill ${ }^{1}$
}

${ }^{1}$ Department of Veterinary and Biomedical Sciences and 2Department of Entomology, The Pennsylvania State University, University Park, Pennsylvania, USA.

\begin{abstract}
Whooping cough is considered a childhood disease, although there is growing evidence that children are infected by adult carriers. Additionally, increasing numbers of vaccinated adults are being diagnosed with Bordetella pertussis disease. Thus it is critical to understand how $B$. pertussis remains endemic even in highly vaccinated or immune populations. Here we used the mouse model to examine the nature of sterilizing immunity to $B$. pertussis. Antibodies were necessary to control infection but did not rapidly clear B. pertussis from the lungs. However, antibodies affected B. pertussis after a delay of at least a week by a mechanism that involved neutrophils and Fc receptors, suggesting that neutrophils phagocytose and clear antibody-opsonized bacteria via Fc receptors. $B$. pertussis blocked migration of neutrophils and inhibited their recruitment to the lungs during the first week of infection by a pertussis toxin-dependent (PTx-dependent) mechanism; a PTx mutant of B. pertussis induced rapid neutrophil recruitment and was rapidly cleared from the lungs by adoptively transferred antibodies. Depletion of neutrophils abrogated the defects of the PTx mutant. Together these results indicate that PTx inhibits neutrophil recruitment, which consequently allows $B$. pertussis to avoid rapid antibody-mediated clearance and therefore successfully infect immune hosts.
\end{abstract}

\section{Introduction}

The widespread use of vaccines in developed nations has decreased the incidence of whooping cough (1-3). However, recent surveys reveal that a majority of individuals in a vaccinated population are transiently infected with the causative agent, Bordetella pertussis, and that it is widespread and endemic (4-8). In vaccinated populations, however, the bacterium induces a mild form of the disease that often goes undiagnosed (9-11). Although severe disease may be the greatest public health concern, undiagnosed pertussis poses an ongoing pervasive risk to very young (pre-vaccine), unvaccinated, and immune-compromised populations. In fact, childhood disease predates the age at which children extensively socialize with each other and appears to commonly have as its source an adult, nonor mildly symptomatic carrier (10-14). The ability of B. pertussis to circulate in vaccinated and immune populations has been known clinically for years but has not been well studied experimentally. Although experimental infection of naive mice may simulate disease, infection of vaccinated or convalescent animals with waning immunity may be more relevant to the biology of the bacterium in a vaccinated population.

Current pertussis vaccines induce a strong serum antibody response that has been shown to be critical for protection from the disease $(15,16)$. However, their efficacy against subclinical infection is doubtful, as the majority of vaccinated populations test positive for subsequent infection $(10,17)$, suggesting that the bacterium successfully infects immune and/or vaccinated individuals. Using animal models, we and others have previously shown that although

Nonstandard abbreviations used: $\mathrm{F} c \gamma \mathrm{R}, \mathrm{F} \gamma \gamma$ receptor; $\mathrm{MH}-\mathrm{S}$, murine alveolar macrophage (cell line); PMN, polymorphonuclear neutrophil; PTx, pertussis toxin; RB6-8C5, anti-Ly-6 monoclonal antibodies.

Conflict of interest: The authors have declared that no conflict of interest exists.

Citation for this article: J. Clin. Invest. 115:3594-3601 (2005).

doi:10.1172/JCI24609.
$B$ cells are necessary for $B$. pertussis clearance from the respiratory tract (18-20), adoptively transferred serum antibodies have little or no effect on bacterial numbers for the first 7 days after inoculation (18-21) but begin to control and clear the bacteria thereafter. The ability to resist rapid antibody-mediated clearance may increase the duration and intensity of infection, both of which facilitate the transmission of the bacteria and would therefore be critical to the endemism of $B$. pertussis in vaccinated populations.

$B$. pertussis is thought to have emerged from a B. bronchisepticalike progenitor $(22,23)$. These closely related subspecies share a similar set of virulence determinants but a different host range (24). Interestingly, while both require B cells for their clearance from the respiratory tract, only B. bronchiseptica is rapidly (within 3 days) cleared by adoptively transferred serum antibodies (20). We previously elucidated the mechanism of antibody-mediated clearance of $B$. bronchiseptica in order to determine the pathway that is presumably inhibited by $B$. pertussis $(25,26)$. Serum antibodymediated clearance of $B$. bronchiseptica requires a TLR4-induced early recruitment of neutrophils that phagocytose bacteria via Fc $\gamma$ receptors ( $F c \gamma R s$ ) and CR3. We hypothesized that serum antibody-mediated clearance of $B$. pertussis also requires neutrophils and that it may resist rapid serum antibody-mediated clearance by inhibiting neutrophil recruitment, presumably via a mechanism not shared by B. bronchiseptica.

Pertussis toxin (PTx), which is only expressed by B. pertussis, is an A-B type toxin known to inhibit $G$ protein signaling pathways that involve Gia, interfering with a class of receptor that includes the majority of the chemokine receptors $(27,28)$. Various in vitro and in vivo studies have demonstrated its ability to inhibit the chemotaxis of neutrophils, lymphocytes, and macrophages (29-31). Although PTx has been proposed to be involved in the pathogenesis of whooping cough (32), its exact role in vivo during $B$. pertussis infection is not yet understood. Addition of inactivated PTx in the pertussis vaccine preparations has helped improve the vaccine's 


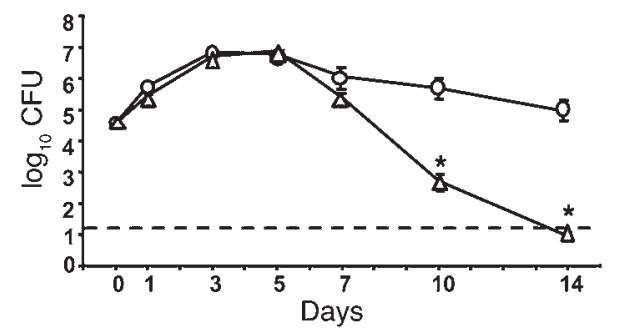

Figure 1

Serum antibodies clear $B$. pertussis after 7 days of inoculation. C57BL/6 mice were inoculated with $5 \times 10^{5} \mathrm{CFU}$ of $B$. pertussis and adoptively transferred with $200 \mu$ l of naive serum (circles) or convalescent-phase serum (triangles). Lungs were harvested on the indicated days, and bacterial numbers were enumerated and expressed as geometric mean \pm SEM. Dotted line denotes limit of detection. $n=4$ per group. ${ }^{*} P<0.001$.

efficacy, suggesting that anti-PTx antibodies are important for protection against $B$. pertussis disease $(15,33)$. Furthermore, a serological study indicated a positive correlation between anti-PTx antibody levels and protection from disease (34).

In the current analysis of the mechanism of antibody-mediated bacterial clearance, we observed that $B$. pertussis clearance was similar to that of B. bronchiseptica: antibody-facilitated clearance of both bacteria required $\mathrm{F} c \gamma \mathrm{Rs}$ and neutrophils. A significant difference, however, was observed in the kinetics of clearance of B. pertussis. The delayed elimination of bacteria correlated with the delayed recruitment of neutrophils to the lungs. Consequently, a PTx mutant of $B$. pertussis was rapidly cleared by adoptively transferred serum antibodies, suggesting that PTx inhibits early neutrophil recruitment and thereby contributes to the delayed antibodymediated bacterial clearance. Expression of PTx may be an adaptation strategy of $B$. pertussis to cause an acute infection and extend its infectious period in immune hosts, facilitating its persistence in immune human populations.

\section{Results}

Serum antibodies do not rapidly clear B. pertussis. We and others have previously shown that $\mathrm{B}$ cells are necessary for the clearance of $B$. pertussis from the lungs (18-20). Therefore we sought to investigate the role of serum antibodies in bacterial clearance. Groups of wild-type mice were inoculated with $5 \times 10^{5} \mathrm{CFU}$ of $B$. pertussis in $50 \mu \mathrm{l}$ of PBS i.n., which has previously been shown to consistently deliver bacteria into the lungs. Immediately after inoculation, $200 \mu \mathrm{l}$ of either naive or convalescent-phase serum were injected i.p. Mice were sacrificed on days $1,3,7,10$, and 14 after inoculation, and lungs were harvested to enumerate the number of bacteria. Numbers of bacteria recovered from the lungs of either naive serum- or convalescent-phase serum-treated mice did not differ significantly on days 1,3 , and 7 after inoculation, suggesting that serum antibodies have minimal effect on bacterial clearance during the first 7 days after inoculation (Figure 1). However, convalescent-phase serum-treated mice harbored only approximately 1,000 bacteria, while naive serum-treated mice harbored approximately $10^{5}$ bacteria on day 10 after inoculation $(P<0.001)$. Similarly, no bacteria could be detected in the lungs of convalescent-phase serum-treated mice on day 14 after inoculation, while approximately $10^{5} \mathrm{CFU}$ of $B$. pertussis were recovered from the lungs of naive serum-treated mice $(P<0.001)$. These data suggest that serum antibodies were able to clear $B$. pertussis from the lungs only after 1 week of infection. Apparently B. pertussis resists the effect of antibodies during the first week of infection, probably by inhibiting 1 or more antibody effector function.

FcyRs, but not C3, are required for serum antibody-mediated clearance of $B$. pertussis. Antibodies may clear bacteria by neutralization, complement-mediated lysis, or opsonization for $\mathrm{F} c \gamma \mathrm{R}$-mediated phagocytosis. We have previously shown that serum antibodies rapidly clear B. bronchiseptica from the lungs of mice and that the mechanism involved both complement and $F_{c \gamma}$ Rs $(25,26)$. Since $B$. pertussis is very closely related to B. bronchiseptica but is cleared much more slowly by serum antibodies, we predicted that $B$. pertussis has some mechanism to resist 1 or more of these antibody effector functions early in the infection. In order to test the importance of complement in serum antibody-mediated bacterial clearance, we used mice lacking the central complement component $\mathrm{C} 3$, required for both classical and alternative complement cascades. Groups of wild-type C57BL/ 6 and congenic $\mathrm{C}^{-/-}$mice were inoculated with $B$. pertussis as described above. Immediately after inoculation, $200 \mu \mathrm{l}$ of naive or $B$. pertussis-induced convalescentphase serum was injected i.p. Fourteen days after inoculation, mice were sacrificed, and lungs were harvested to enumerate $B$. pertussis CFU. Naive serum had no effect on bacterial numbers in the lungs of wild-type or $\mathrm{C}^{-/-}$mice (Figure 2 ). Convalescent-phase serum completely cleared the bacteria from the lungs of wild-type and $\mathrm{C}^{-/-}$mice by day $14(P<0.001)$. These data indicate that $\mathrm{C} 3$ is not required for serum antibody-mediated clearance of $B$. pertussis.

In order to test the importance of Fc $\gamma$ Rs in antibody-mediated clearance of $B$. pertussis, we used $\mathrm{Fc} \mathrm{R}^{-/-}$mice, which lack all $3 \mathrm{Fc}$ receptors for $\operatorname{IgG}$ and 1 for $\operatorname{IgE}(35)$. Groups of $\mathrm{Fc} \gamma \mathrm{R}^{-/-}$mice were inoculated with $B$. pertussis and adoptively transferred naive serum or convalescent-phase serum as described above. As opposed to wild-type mice, in which convalescent-phase serum completely cleared bacteria from the lungs within 14 days, the same immune serum had no effect on bacterial numbers in the lungs of $\mathrm{Fc}_{\mathrm{C}} \mathrm{R}^{-1}$ mice $(P<0.001$; Figure 2$)$. These data indicate that Fc $\gamma$ Rs are required for serum antibody-mediated clearance of $B$. pertussis. Together, these data showed that antibodies were not functioning by neutralization alone or via complement-mediated lysis. Antibodies appeared to clear $B$. pertussis from the lungs via phagocytosis by recruited Fc $\gamma$ R-bearing cells.

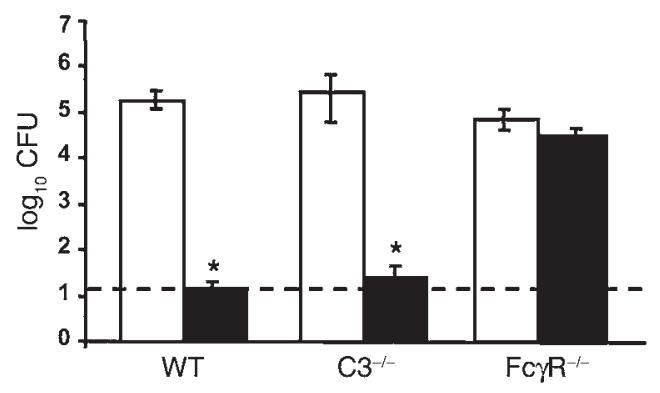

Figure 2

Adoptively transferred antibodies clear $B$. pertussis in the lungs of wild-type and $\mathrm{C}^{-/-}$, but not $\mathrm{Fc}_{\mathrm{R}} \mathrm{R}^{-1}$, mice. C57BL/6 (WT), $\mathrm{C}^{-/-}$, and $\mathrm{Fc} \gamma \mathrm{R}^{-1-}$ mice were inoculated with $5 \times 10^{5} \mathrm{CFU}$ of $B$. pertussis and adoptively transferred $200 \mu \mathrm{l}$ of either naive serum (white bars) or convalescent-phase serum (black bars). On day 14 after inoculation, mice were sacrificed, and bacteria in the lungs were enumerated and expressed as geometric mean \pm SEM. Dotted line denotes limit of detection. $n=4$ per group. ${ }^{\star} P<0.001$. 


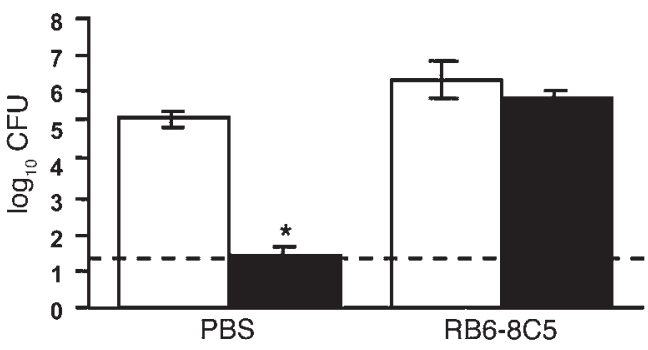

\section{Figure 3}

Depletion of neutrophils abrogates antibody-mediated clearance of B. pertussis. C57BL/6 mice were treated with PBS or $1 \mathrm{mg}$ of neutrophil-depleting mAb RB6-8C5. Mice were subsequently inoculated with $5 \times 10^{5} \mathrm{CFU}$ of $B$. pertussis and adoptively transferred $200 \mu \mathrm{l}$ of either naive (white bars) or convalescent-phase serum (black bars). On day 14 after inoculation, mice were sacrificed, and bacteria in the lungs were enumerated and expressed as geometric mean \pm SEM. Dotted line denotes limit of detection. $n=4$ per group. ${ }^{*} P<0.001$.

Neutrophils are required for antibody-mediated clearance of B. pertussis. We have previously shown that Fc $\gamma \mathrm{R}$-bearing neutrophils are the primary inflammatory cells recruited to the lungs following infection with $B$. bronchiseptica and are required for rapid antibodymediated bacterial clearance $(26,36)$. Since $B$. pertussis clearance required FcyRs, we sought to determine whether neutrophils are required for antibody-mediated clearance of this organism. Groups of C57BL/ 6 mice were injected i.p. with PBS or $1 \mathrm{mg}$ antiLy-6 monoclonal antibodies (RB6-8C5). This treatment has been previously shown to deplete neutrophils for at least 2 weeks with no apparent effect on other cells such as macrophages or dendritic cells $(37,38)$. Following treatment, mice were inoculated with $B$. pertussis and adoptively transferred naive or immune serum as described above. The mice were sacrificed on day 14 after inoculation, and bacterial numbers in the lungs were enumerated (Figure $3)$. Naive serum had no effect on bacterial numbers in the lungs of mice treated with PBS or RB6-8C5. However, immune serum completely cleared bacteria from the lungs of mice treated with PBS, but not those treated with RB6-8C5 $(P<0.001)$, indicating that neutrophils are required for antibody-mediated bacterial clearance. The above-mentioned observations were verified using a recently isolated strain of B. pertussis, 6068 (a kind gift from Jeff Miller, UCLA, Los Angeles, California, USA).

$P T x$ inhibits antibody-mediated clearance of B. pertussis. B. bronchiseptica and $B$. pertussis are very closely related and share a majority of the known virulence genes $(22,23)$. These 2 closely related subspecies both required similar antibody effector functions and phagocytic cells for their clearance from the lungs of mice (Figure 2 and ref. 26). Yet immune serum substantially reduces the numbers of $B$. bronchiseptica in the lungs within 1 day (by more than $99 \%$ ), whereas it has no effect on the numbers of $B$. pertussis for at least 7 days after inoculation (20). These data suggested that some $B$. pertussis-specific virulence gene(s) delay antibody-mediated bacterial clearance. PTx is a $B$. pertussis-specific virulence factor that has been extensively studied in vitro and shown to inhibit the downstream effects of $G$ protein-coupled receptors, including many chemokine receptors $(27,28)$. Furthermore, in vitro and in vivo experiments using purified PTx have shown that PTx can inhibit recruitment of cells such as neutrophils and lymphocytes (29-31). One plausible explanation for the delayed bacte- rial clearance may be that secreted PTx inhibits early neutrophil recruitment, which is necessary for antibody-mediated clearance. In support of this argument, we have previously observed that $B$. bronchiseptica induces significantly more neutrophil infiltration than B. pertussis on day 3 after inoculation (36). Therefore, we predicted that in the absence of $\mathrm{PTx}, B$. pertussis would be more susceptible to antibody-mediated clearance. In order to test this hypothesis, we inoculated mice with $5 \times 10^{5} \mathrm{CFU}$ of $B$. pertussis or the PTx mutant $B$. pertussis $\triangle P T x$ as described above and adoptively transferred either naive or convalescent-phase serum. Lungs were harvested from these mice on days 3 and 7 after inoculation, and bacterial numbers in the lungs were enumerated. Convalescentphase serum rapidly reduced numbers of $B$. pertussis $\triangle P T x$ in the lungs as early as 3 days after inoculation $(P<0.05)$ and completely cleared this bacterium within 7 days $(P<0.05)$, but had no effect on the numbers of $B$. pertussis in this time frame (Figure 4$)$. This was not due to the higher number of wild-type bacteria, since we have previously observed that adoptively transferred antibodies clear more than $10^{6}$ bacteria when PTx is not present (G.S. Kirimanjeswara and E.T. Harvill, unpublished observations). These data suggest that PTx is involved in inhibiting antibody-mediated clearance of $B$. pertussis, perhaps by inhibiting neutrophil migration to the lungs. Additionally, analysis of the antibody-mediated clearance of $B$. pertussis in the trachea indicated a similar mechanism as that of the lungs was involved, and PTx inhibited rapid bacterial clearance in this organ as well. However, no significant effect of adoptively transferred serum antibodies was observed on the bacterial numbers in the nasal cavity on any tested days.

$P T x$ reduces neutrophil recruitment to the lungs. B. bronchiseptica induces the recruitment to the lungs of substantially higher numbers of neutrophils than does $B$. pertussis during the first week of infection $(20,26,36)$. Since neutrophils are required for antibody-mediated clearance of both B. bronchiseptica and B. pertussis, we predicted that $B$. pertussis has mechanisms to inhibit neutrophil recruitment to the lungs in order to resist the effect of serum antibodies. Additionally, as previous studies have shown that PTx reduces the proportion of neutrophils recovered in the broncheoalveolar lavage fluid (39), we hypothesized that PTx inhibits the migration of neutrophils to the lungs, decreasing their num-

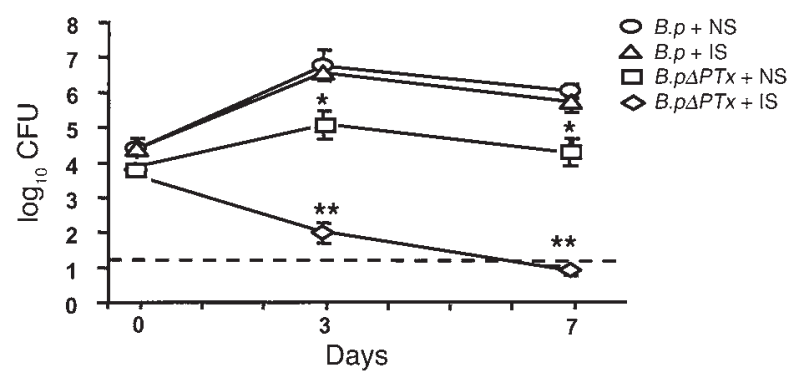

\section{Figure 4}

Serum antibodies rapidly clear $B$. pertussis $\triangle P T x$ but not $B$. pertussis. C57BL/6 mice were inoculated with either B. pertussis (B.p) or $B$. pertussis $\triangle P T X(B . p \triangle P T X)$. Subsequently, these mice were adoptively transferred $200 \mu$ l of either naive serum (NS) or convalescentphase serum (IS). Mice were sacrificed on the indicated days, and bacterial numbers in the lungs were determined and expressed as geometric mean \pm SEM. Dotted line denotes limit of detection. $n=4$ per group. ${ }^{\star} P<0.001 ;{ }^{*} P<0.05$. 


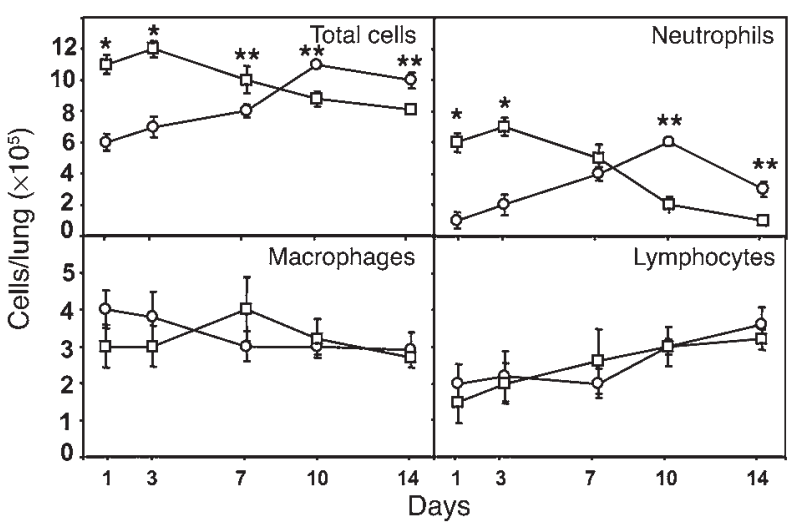

Figure 5

PTx inhibits recruitment of neutrophils to the lungs. C57BL/6 mice were inoculated with $5 \times 10^{5} \mathrm{CFU}$ of $B$. pertussis (circles) or $B$. pertussis $\triangle P T x$ (squares). Mice were sacrificed on the indicated days and their lungs perfused with $5 \mathrm{ml}$ cold PBS, and total leukocytes per whole lung were enumerated. Individual cell types were determined by modified Giemsa staining of cells. $n=4$ per group. ${ }^{\star} P<0.001 ;{ }^{\star \star} P<0.05$.

bers and thereby inhibiting antibody-mediated bacterial clearance. To test these hypotheses, groups of mice were inoculated with $B$. pertussis or $B$. pertussis $\triangle P T x$ as described above, and leukocytes recruited to the lungs were enumerated. Approximately $5-6 \times 10^{5}$ leukocytes were observed on day 1 after inoculation, and $7-8 \times 10^{5}$ on days 3 and 7 , in the lungs of mice inoculated with $B$. pertussis (Figure 5). On days 1, 3, and 7 after inoculation, the majority of leukocytes in the lungs of mice inoculated with $B$. pertussis were macrophages $\left(\sim 4 \times 10^{5}\right)$, a small percentage of cells were lymphocytes $\left(\sim 2 \times 10^{5}\right)$, and the rest were neutrophils $\left(\sim 1 \times 10^{5}\right)$. In contrast, the lungs of mice inoculated with $B$. pertussis $\triangle P T x$ harbored approximately $10-12 \times 10^{5}$ leukocytes on days 1,3 and 7 after inoculation, and the majority of these cells were neutrophils $\left(5-6 \times 10^{5}\right)$. The absolute number of macrophages and lymphocytes recruited were similar between the 2 groups $(P<0.05)$. These data indicate that the higher proportion of neutrophils previously reported to be recruited by $B$. pertussis $\triangle P T X$ (39) may be attributed to a large increase in absolute numbers of neutrophils within the lungs. Thus, we concluded that PTx appears to inhibit neutrophil recruitment to the lungs during the first week of infection. As reported by other investigators (40), the number of leukocytes, particularly neutrophils, began to increase in the lungs of mice infected with $B$. pertussis on day 7 after inoculation, reaching a peak at day 10 after inoculation. On day 10 , the lungs of mice inoculated with $B$. pertussis harbored approximately $10 \times 10^{5}$ leukocytes, of which $5-6 \times 10^{5}$ cells were neutrophils and $2-3 \times 10^{5}$ were lymphocytes, indicating that the effects of PTx are ultimately overcome and neutrophils are recruited to the lungs later during infection. Interestingly, this delayed neutrophil recruitment correlates with the time frame in which antibodies begin to be effective against $B$. pertussis.

Antibody-mediated clearance of $B$. pertussis $\triangle P T x$ requires $F c \gamma R$ s and neutrophils. The above data reveal a strong correlation between neutrophil recruitment to the lungs and serum antibody-mediated bacterial clearance and suggest that neutrophils eliminate $B$. pertussis via Fc $\gamma$ R-mediated opsonization and phagocytosis. However, it is possible that $B$. pertussis $\triangle P T X$ may be cleared by serum antibodies by a mechanism different from that of wild-type $B$. pertussis. There- fore, we sought to determine the mechanism of antibody-mediated clearance of B. pertussis $\triangle P T x$. Groups of C57BL/6 and $\mathrm{Fc} \gamma \mathrm{R}^{-/-}$mice were inoculated with $B$. pertussis $\triangle P T x$ and adoptively transferred $200 \mu \mathrm{l}$ of either naive or immune serum as described above. Naive serum had no significant effect on the bacterial numbers in the lungs of wild-type or $\mathrm{Fc}_{\mathrm{R}} \mathrm{R}^{-/}$mice on either day 3 or day 7 (Figure $6 \mathrm{~A})$. In wild-type mice, immune serum rapidly reduced bacterial numbers in the lungs by day 3 after inoculation $(P<0.001)$ and completely cleared bacteria by day $7(P<0.001)$. However, no significant reduction in the numbers of bacteria was observed in the lungs of immune serum-treated $\mathrm{Fc} \gamma \mathrm{R}^{-/-}$mice on days 3 and 7 after inoculation, indicating that $\mathrm{Fc} \gamma \mathrm{Rs}$ are required for antibody-mediated clearance of $B$. pertussis $\triangle P T x$.

Based on these results, we tested the hypothesis that neutrophils are involved in rapid antibody-mediated clearance of B. pertussis $\triangle P T x$. Groups of C57BL/6 mice were inoculated with B. pertussis $\triangle P T x$ and adoptively transferred $200 \mu$ l of either naive or immune serum as described above. Half of the mice in each group were also given $1 \mathrm{mg}$ RB6-8C5 injected i.p. to deplete neutrophils, and the other half were given PBS as control. Among the mice given naive serum, RB6-8C5 treatment resulted in approximately 10 - to 100 -fold higher numbers of $B$. pertussis $\triangle P T X$ in the lungs compared with PBS treatment (Figure 6B). These numbers were indistinguishable from the numbers of wild-type $B$. pertussis in the lungs at this time point (compare to Figure 4), indicating that PTx is not required for efficient colonization when neutrophils are depleted. These data strongly suggest that PTx enables $B$. pertussis to colonize the lung by inhibiting neutrophil recruitment, as proposed by Carbonetti et al. (39). Immune serum rapidly reduced the number of $B$. pertussis $\triangle P T x$ in the lungs of mice treated with PBS on day $3(P<0.001)$ and completely cleared bacteria on day 7 after inoculation $(P<0.001)$. However, immune serum failed to reduce the number of bacteria on days 3 and 7 after inocula-

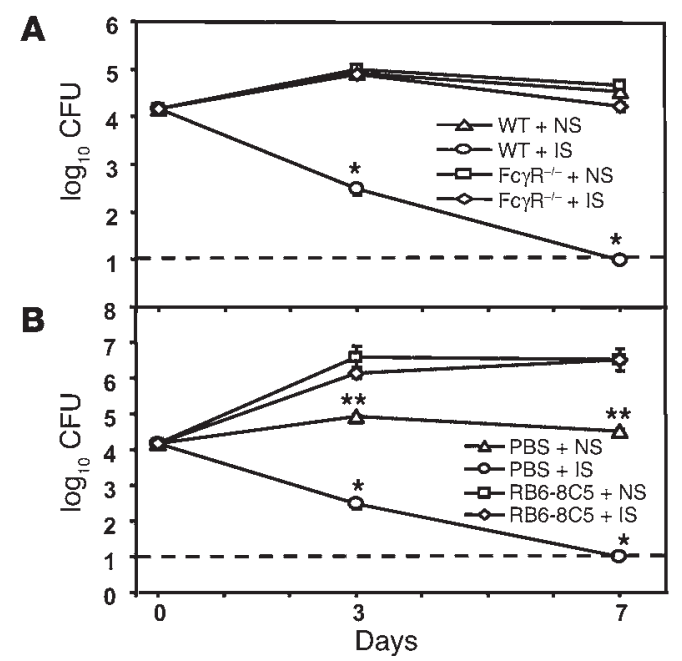

Figure 6

F c $\gamma$ Rs and neutrophils are required for serum antibody-mediated clearance of $B$. pertussis $\triangle P T x$. (A) C57BL/6 or $\mathrm{Fc}_{\gamma} \mathrm{R}^{-1-}$ mice were inoculated with $5 \times 10^{5} \mathrm{CFU}$ of $B$. pertussis $\triangle P T x$ and adoptively transferred $200 \mu$ l of either naive or convalescent-phase serum. (B) C57BL/6 mice also received either PBS or $1 \mathrm{mg}$ of mAb RB6-8C5 ( $n=4$ per group). Lungs were harvested on the indicated days, and bacteria were enumerated and expressed as mean \pm SEM. Dotted line denotes limit of detection. $n=4$ per group. ${ }^{\star} P<0.001 ;{ }^{* \star} P<0.05$. 


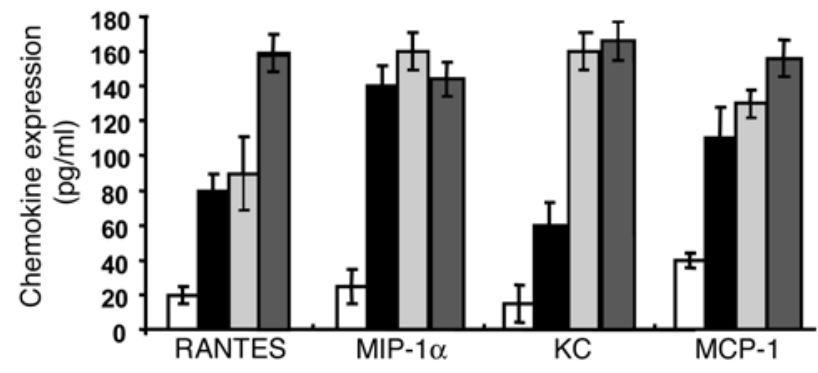

Figure 7

PTx does not modulate chemokine production by alveolar macrophages. $\mathrm{MH}-\mathrm{S}$ cells were incubated with $B$. pertussis (black bars) or $B$. pertussis $\triangle P T x$ (dark gray bars) at $\mathrm{MOI}$ of 10 for 12 hours. Also shown are medium controls (white bars) and positive controls $(100 \mathrm{ng} / \mathrm{ml}$ E. coli LPS, light gray bars). Culture supernatants were collected and analyzed for chemokines by ELISA and expressed as mean \pm SEM.

tion in mice treated with RB6-8C5, indicating that neutrophils are required for rapid serum antibody-mediated clearance of $B$. pertussis $\triangle P T X$. These data indicate that serum antibodies clear both $B$. pertussis and $B$. pertussis $\triangle P T x$ by a similar mechanism involving Fc $\gamma$ R-dependent phagocytosis by neutrophils. These data were verified using an independently created PTx mutant of B. pertussis (a kind gift from N. Carbonetti, University of Maryland, College Park, Maryland, USA).

PTX does not modulate chemokine and cytokine production by alveolar macrophages. PTx could affect neutrophil recruitment in various ways. It is known to influence chemokine and cytokine responses (41), interfere with $\mathrm{G}$ protein-coupled chemokine receptor signaling (42), and downregulate adhesion molecule expression on endothelial cells (43). To investigate whether PTx modulates chemokine expression by alveolar macrophages, a confluent layer of murine alveolar macrophage (MH-S) cells was incubated with $B$. pertussis or B. pertussis $\triangle P T x$ at an MOI of 10 for 12 hours. The accumulated amounts of various chemokines, such as macrophage inflammatory protein-1 $\alpha$ (MIP-1 $\alpha)$, monocyte chemoattractant protein 1 (MCP-1), chemokine RANTES (RANTES), and chemokine $\mathrm{KC}(\mathrm{KC})$, were then analyzed by ELISA. Figure 7 shows that macrophages produced significant amounts of these chemokines when treated with $100 \mathrm{ng} / \mathrm{ml}$ of E. coli LPS as a positive control or infected with $B$. pertussis or $B$. pertussis $\triangle P T x$. However, no significant differences were observed between the number of chemokines produced by macrophages incubated with these 2 strains. These data suggest that PTx does not inhibit neutrophil recruitment by blocking chemokine production.

PTx inhibits neutrophil migration. In order to determine whether PTx acts directly on neutrophils or endothelial cells to inhibit neutrophil recruitment to the lungs, we used an in vitro neutrophil migration assay to determine the number of neutrophils that migrate across endothelial cells toward chemokines produced by macrophages incubated with either $B$. pertussis or B. pertussis $\triangle P T x$ at an MOI of 10 for 12 hours. The average number of neutrophils per field that migrated toward a media negative control was approximately 35 (Figure 8). More than 500 neutrophils per field were found to migrate across an endothelial cell barrier toward the supernatant from macrophages treated with E. coli LPS. The number of neutrophils that migrated toward supernatant from macrophages incubated with $B$. pertussis was slightly higher than the nega- tive control at approximately 45 per field. However, the number of neutrophils that migrated toward supernatant from macrophages incubated with $B$. pertussis $\triangle P T x$ was approximately 400 per field $(P<0.001)$. Since our previous data indicated that PTx does not modulate chemokine production by alveolar macrophages, we predicted that PTx in supernatants from macrophages incubated with $B$. pertussis may alter chemotaxis of neutrophils. In order to address this possibility, equal amounts of supernatants from cells incubated with $B$. pertussis and $B$. pertussis $\triangle P T x$ were mixed and used for the in vitro neutrophil migration assay. The number of neutrophils that migrated toward this mixture was approximately 50 per field, similar to the number of neutrophils that migrated toward supernatant from cells incubated with $B$. pertussis alone. These data suggest that culture supernatants from cells incubated with $B$. pertussis have some components that inhibit chemotaxis of neutrophils toward supernatant from $B$. pertussis $\triangle P T x$-exposed macrophages.

Since PTx has been shown to affect $G$ protein-coupled receptor signaling pathways that may influence chemotaxis, we hypothesized that the PTx present in the supernatant from macrophages incubated with $B$. pertussis may directly act on neutrophils to decrease their migration across endothelial cells. To test this, we mixed various amounts of purified PTx (obtained from N. Carbonetti) with the supernatant from macrophages incubated with $B$. pertussis $\triangle P T x$. Neutrophil migration toward supernatants from cells incubated with $B$. pertussis $\triangle P T x$ was reduced by $90 \%$ when purified PTx was added $(P<0.001$; Figure 8$)$. However, adding the same amount of catalytically inactivated PTx (a kind gift of N. Carbonetti; described in refs. 39, 44-46) had no effect on the migration of neutrophils across endothelial cells. Mixing purified PTx with supernatant from macrophages treated with E. coli LPS also resulted in a significant reduction in neutrophil migration $(P<0.05)$. These data indicate that PTx inhibits the migration of neutrophils across endothelial cells and that this inhibition is dependent on its enzymatic activity.

The inhibitory effect of PTx may be due to its action on endothelial cells or on neutrophils. In order to differentiate between

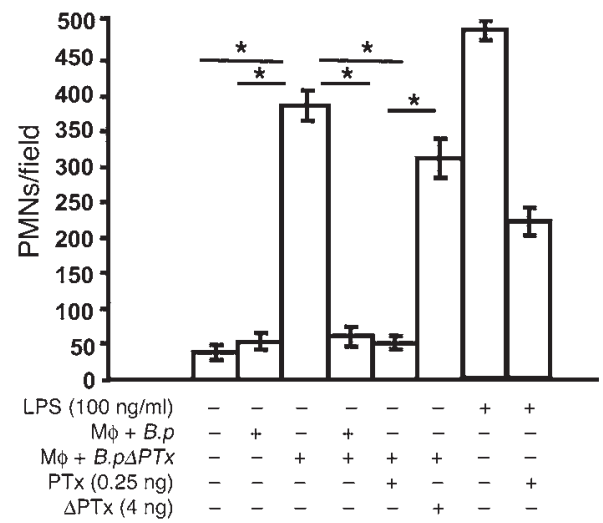

Figure 8

PTx inhibits neutrophil migration. In 3- $\mu \mathrm{M}$ transwell chambers, $10^{5}$ PMNs were allowed to migrate across endothelial cells toward supernatant from alveolar macrophages $(\mathrm{M} \phi)$ incubated with $B$. pertussis or $B$. pertussis $\triangle P T x$. Purified PTx or catalytically inactive PTx $(\triangle \mathrm{PTx})$ were added to the supernatant as indicated. The number of neutrophils that migrated to the lower chamber were enumerated by observing 10 fields and expressed as mean \pm SEM. The experiments were repeated at least 3 times. ${ }^{*} P<0.001$. 
these 2 possibilities, endothelial cells growing on the upper chamber of the transwell system were preincubated with $B$. pertussis or $B$. pertussis $\triangle P T x$. Subsequently, neutrophils were allowed to migrate across them toward supernatants from macrophages incubated with $B$. pertussis $\triangle P T x$. Preincubation of endothelial cells with either $B$. pertussis or $B$. pertussis $\triangle P T x$ did not alter neutrophil migration (data not shown). In fact, neutrophil migration assays carried out in the absence of endothelial cells showed that adding PTx to supernatant from macrophages incubated with $B$. pertussis $\triangle P T x$ inhibited migration of neutrophils (data not shown). These data suggest that PTx acts directly on neutrophils and affects their chemotaxis across a membrane and their migration across endothelial cells.

\section{Discussion}

PTx has been studied extensively for its adjuvant properties (47) and inhibition of GTPase activities $(48,49)$. Although it has many effects on various cell types in vitro, its contributions to $B$. pertussis infection and pathogenesis have not yet been determined. Recently, Carbonetti et al. reported that PTx is required for efficient early colonization by $B$. pertussis in the lungs of mice (39). Our present data indicate that PTx facilitates $B$. pertussis colonization by inhibiting early recruitment of neutrophils to the lungs. However, we also noted a much stronger effect of PTx on the ability of B. pertussis to resist antibody-mediated clearance that may be critical to the biology of this endemic human pathogen.

Based on its known in vitro activities, PTx could inhibit neutrophil recruitment by modulating chemokine or cytokine production by epithelial cells and macrophages, directly interfering with the chemokine receptor signaling, and/or altering adhesion molecule expression that may interfere with the diapedesis of blood leukocytes $(30,41,45,50,51)$. In our study, both $B$. pertussis and $B$. pertussis $\triangle P T x$ induced production of similar levels of a range of chemokines by alveolar macrophages. However, only the supernatant from macrophages incubated with $B$. pertussis $\triangle P T x$ facilitated the migration of neutrophils across endothelial cells. Additionally, purified PTx, but not catalytically inactive PTx, was able to inhibit the migration of neutrophils in this assay. Together, these data indicate that PTx inhibits polymorphonuclear neutrophil (PMN) recruitment by acting directly on chemokine receptor signaling of neutrophils. However, other effects of PTx may also contribute to the inhibition of neutrophil recruitment in vivo. The migration of neutrophils across airway epithelium requires PTx-sensitive signals (52), but the nature of these signals is difficult to delineate during an active infection with $B$. pertussis, not only due to the multiple possible inhibitory effects of PTx, but also due to the variety of pro- and antiinflammatory factors induced and released by the bacteria. Interestingly, the number of neutrophils recruited to the lungs increased after day 7 after inoculation in spite of the presence of PTx. This increase in the number of neutrophils correlates with the kinetics of antibody-mediated bacterial clearance. We have observed that a $\mathrm{T}$ cell-dependent response was required to overcome the effect of PTx and allow antibody-mediated bacterial clearance during the second week of infection (data not shown). The implication of an inhibitory effect of PTx on neutrophil recruitment is 2 -fold: it allows $B$. pertussis to evade innate immunity early in colonization and also provides a means to resist the effect of serum antibodies in the lungs. Although the former effect may be important to primary infection in naive, unvaccinated hosts, the latter may be more relevant to the persistence of $B$. pertussis as an endemic pathogen even within vaccinated populations.
Acute, highly contagious, immunizing pathogens face the significant epidemiological challenge of long-term persistence within the host population. Immunity results in depletion of susceptible hosts through the course of each epidemic; thereafter host replenishment requires births of susceptible individuals or loss of immunity - which is why pathogens that convey strong, long-lasting immunity result in "childhood diseases" (53). Rapid contagion, in turn, results in fast transmission among hosts, which is a shortterm evolutionary benefit to the pathogen $(32,54)$. However, it also results in large-amplitude epidemics with intervening deep epidemic troughs. In small and medium host populations, the chain of transmission will be broken in the troughs so that the pathogen will go extinct. The most relevant theoretical models for childhood infections, the so-called realistic age-structured models $(55,56)$, predict an endemic threshold of around half a million hosts in order for transmission to be sustained through the epidemic troughs of acute, immunizing infections. This prediction is closely matched by epidemiological surveillance data $(57,58)$. Previous theoretical studies have highlighted 2 key adaptations that increase the height of the epidemic troughs to allow long-term endemism within smaller host communities: (a) reinfection of previously immunized hosts and adult carriers and (b) prolongation of the infectious period (7). Our study is of wide epidemiological significance in showing that B. pertussis, through expression of PTx, slows migration of neutrophils and thereby extends the infection period (relative to $B$. pertussis $\triangle P T x$ strains) and allows for transient reinfection of previously immunized hosts. PTx expression may, therefore, be a key adaptation by $B$. pertussis for interacting with the unique population dynamics of its human host.

Susceptibility of humans to B. pertussis infection, particularly among people with no history of disease, has been shown to correlate with low levels of anti-PTx antibodies (59). Other studies have recognized that PTx is one of the critical components necessary for higher efficacy of acellular pertussis vaccines $(34,60)$. Although antibody titer to pertactin positively correlates with the resistance to diagnosed disease symptoms, addition of pertactin or filamentous hemagglutinin to PTx increases the efficacy of acellular pertussis vaccines $(15,33)$. Interestingly, antibody titers to PTx decrease much faster than those to other $B$. pertussis antigens (61). Since PTx allows B. pertussis to largely avoid the effects of antibodies to other antigens, the most effective vaccination strategy to $B$. pertussis may involve an increased focus on the induction of a long-lasting serum antibody response to PTx. Use of genetically inactivated mutant PTx in the current acellular vaccines, as proposed by Robbins et al. (62), may be a substantial improvement in achieving higher level of anti-PTx antibodies that reduce the rate of infection and severity of disease.

\section{Methods}

Bacteria. B. pertussis strain BP536 is a streptomycin-resistant derivative of Tohama I (36). BPH101 (B. pertussis $\triangle P T x$ ) is a PTx mutant of BP536 and was a kind gift from D. Burns (FDA, Rockville, Maryland, USA) (63). Bacteria were maintained on Bordet-Gengou (BG) agar (BD Diagnostics) supplemented with 7.5\% defibrinated sheep's blood (Remel or Hema Resource \& Supply Inc.) and $20 \mu \mathrm{g} / \mathrm{ml}$ streptomycin. Bacteria were grown in Stainer-Scholte (SS) broth with supplements and $20 \mu \mathrm{g} / \mathrm{ml}$ streptomycin to mid-log phase (optical densities of approximately 0.3 at $600 \mathrm{~nm}$ ) at $37^{\circ} \mathrm{C}$ on a roller drum for experiments. Genetically inactivated PTx was a gift from N. Carbonetti and has been described previously (44-46). This mutant PTx has 2 amino acid changes: Arg 9 to Lys and 
Glu 129 to Gly. This mutant PTx has been shown to be immunogenic but not toxigenic.

Inoculation and adoptive transfer protocols. C57BL/6 mice were obtained from Jackson ImmunoResearch Laboratories, and $\mathrm{C}^{-/-}$mice backcrossed extensively onto a C57BL/6 background have been described previously (64) and were kind gifts of R. Wetsel (University of Texas - Houston, Houston, Texas, USA). Fc $\gamma \mathrm{R}^{-/-}$(C57BL/6 background) were obtained from Taconic and have been described previously (35). Mice were lightly sedated with isoflurane (IsoFlo; Abbott Laboratories), and $5 \times 10^{5} \mathrm{CFU}$ of bacteria in 50 $\mu \mathrm{l}$ of PBS were inoculated onto the tips of the external nares. Colonization levels were determined by homogenizing the lungs in $1 \times$ PBS and plating aliquots for colony counts. The homogenates and necessary dilutions were plated in 50- $\mu 1$ volumes onto BG agar with streptomycin. Colonies were counted after 3 days' incubation at $37^{\circ} \mathrm{C}$. For adoptive transfer experiments, mice were inoculated with $B$. pertussis or $B$. pertussis $\triangle P T X$ as described above, immediately followed by i.p. injection of $200 \mu \mathrm{l}$ convalescent-phase serum obtained from BP536-inoculated mice on day 28 after inoculation. Various batches of convalescent-phase sera were tested for anti-B. pertussis antibody levels and used if they matched the titers described by Kirimanjeswara et al. (20). Animals were sacrificed on the indicated day after transfer (Figure Legends 1-6), and bacterial numbers were determined as described above. Animals were handled in accordance with institutional guidelines of The Pennsylvania State University. All animal experiments were approved by the Institutional Animal Care and Use Committee of The Pennsylvania State University. Statistical significance of data points was determined using a 2-tailed Student's unpaired $t$ test.

Enumeration ofleukocytes in the lungs. Total leukocytes were isolated from the lungs after collagenase type I and DNAse I digestion as described previously (65). Briefly, lungs were perfused with PBS and finely sheared with scissors. This lung homogenate was subjected to collagenase type I and DNAse I treatment for about 3 hours. The enzymatically treated homogenate was laid over Histopaque 1119 (Sigma-Aldrich) and centrifuged for 30 minutes at $1,200 \mathrm{~g}$. The leukocyte portion was collected, and the total number of cells was determined by hemocytometer. Individual cell types were determined by staining the isolated cells with modified Giemsa stain by a certified clinical laboratory technician.

Estimation of cytokines and chemokines. MH-S cells (66) were obtained from ATCC and cultured in DMEM medium supplemented with 10\% FBS. Cells were grown to confluency and incubated with either unwashed B. pertussis or $B$. pertussis $\triangle P T x$ at $\mathrm{MOI}$ of 10 for 12 hours at $37^{\circ} \mathrm{C}$. Culture supernatants were collected, filter sterilized, and stored at $-80^{\circ} \mathrm{C}$ for further use. Concentrations of KC, RANTES, MIP-1 $\alpha$, and MCP- 1 were estimated by ELISA.
The percentage of cell death was measured by lactate dehydrogenase assay and was below $15 \%$ at the tested MOI for both strains of bacteria.

Neutrophil migration assay. Primary murine aortic endothelial cells $\left(10^{5}\right.$ cells; a kind gift from L. Sordillo, Michigan State University, East Lansing, Michigan, USA) were cultured as a confluent monolayer on the upper chamber of 3- $\mu \mathrm{M}$ transwells with DMEM supplemented with $10 \%$ FCS for 24 hours. Peripheral blood PMNs were collected from C57BL/ 6 mice by differential density separation using Histopaque 1119 and 1077 (67) and resuspended in DMEM supplemented with 10\% FCS. The percentage of PMNs in the cell suspension was estimated by Giemsa staining of isolated cells and was found to be approximately $90 \%$. Approximately $10^{5} \mathrm{PMNs}$ in a total volume of 200 $\mu l$ of DMEM were layered on the endothelial cells of the upper chamber of the transwell system. Two hundred microliters of supernatant from macrophages treated with $100 \mathrm{ng} / \mathrm{ml}$ of E. coli LPS or cultured with B. pertussis or B. pertussis $\triangle P T x$ for 12 hours at MOI of 1:10 was used as a source of chemoattractants in the lower chamber of the transwell system. After 12 hours, the number of neutrophils that migrated to the lower chamber was measured by observing 10 random fields of the lower chamber under a light microscope.

Neutrophil depletion. RB6-8C5 is a mAb raised against Ly-6 present on neutrophils and was a kind gift from G. Huffnagle (University of Michigan Medical School, Ann Arbor, Michigan, USA) (68). Previously, $1 \mathrm{mg}$ of this $\mathrm{mAb}$ injected i.p. has been shown to deplete neutrophils for 7-14 days (37, 38). Peripheral PMNs were enumerated to determine the efficacy of the treatment and was below $2 \%$.

\section{Acknowledgments}

We thank all the members of E.T. Harvill's lab, Avery August, Andrew Henderson, and the members of the Center for Infectious Disease Dynamics, The Pennsylvania State University, for critical reading of this manuscript and discussion of this study. We thank Nicholas Carbonetti (University of Maryland), Jeff Miller (UCLA), and Drusilla Burns (FDA) for providing various strains of $B$. pertussis and purified PTx. This work was supported by NIH grant 5-R01-A1053075-02.

Received for publication January 31, 2005, and accepted in revised form September 20, 2005.

Address correspondence to: Eric T. Harvill, 115 Henning Building, The Pennsylvania State University, University Park, Pennsylvania 16802, USA. Phone: (814) 863-8522; Fax: (814) 863-6140; E-mail: eth10@psu.edu.
1. Cherry, J.D. 1984. The epidemiology of pertussis and pertussis immunization in the United Kingdom and the United States: a comparative study. Curr. Probl. Pediatr. 14:1-78.

2. Cherry, J.D., Brunell, P.A., Golden, G.S., and Karson, D.T. 1988. Report of the task force on pertussis and pertussis immunization-1988. Pediatrics. 81(Suppl.):939-984.

3. Cherry, J.D., and Heininger, U. 2004. Pertussis and other Bordetella infections. In Textbook of pediatric infectious diseases. W.B. Saunders. Philadelphia, Pennsylvania, USA. 1588-1608.

4. Gilberg, S., et al. 2002. Evidence of Bordetella pertussis infection in adults presenting with persistent cough in a French area with very high whole-cell vaccine coverage. J. Infect. Dis. 186:415-418.

5. Hodder, S.L., et al. 2000. Antibody responses to Bordetella pertussis antigens and clinical correlations in elderly community residents. Clin. Infect. Dis. 31:7-14.

6. Nennig, M.E., Shinefield, H.R., Edwards, K.M., Black, S.B., and Fireman, B.H. 1996. Prevalence and incidence of adult pertussis in an urban population. JAMA. 275:1672-1674.

7. Schmitt-Grohe, S., et al. 1995. Pertussis in German adults. Clin. Infect. Dis. 21:860-866.

8. Wright, S.W., Edwards, K.M., Decker, M.D., and Zeldin, M.H. 1995. Pertussis infection in adults with persistent cough. JAMA. 273:1044-1046.

9. Deville, J.G., et al. 1995. Frequency of unrecognized Bordetella pertussis infections in adults. Clin. Infect. Dis. 21:639-642.

10. Deen, J.L., et al. 1995. Household contact study of Bordetella pertussis infections. Clin. Infect. Dis. 21:1211-1219.

11. Cherry, J.D., et al. 1995. Comparison of values of antibody to Bordetella pertussis antigens in young German and American men. Clin. Infect. Dis. 20:1271-1274.

12. Crowcroft, N.S., et al. 2003. Severe and unrecognised: pertussis in UK infants. Arch. Dis. Child. 88:802-806.

13. Baron, S., et al. 1998. Epidemiology of pertussis in French hospitals in 1993 and 1994: thirty years after a routine use of vaccination. Pediatr. Infect. Dis. J. 17:412-418.

14. Bisgard, K.M., et al. 2004. Infant pertussis: who was the source? Pediatr. Infect. Dis. J. 23:985-989.

15. Cherry, J.D., Gornbein, J., Heininger, U., and Stehr, K. 1998. A search for serologic correlates of immunity to Bordetella pertussis cough illnesses. Vaccine. 16:1901-1906.

16. Storsaeter, J., Hallander, H.O., Gustafsson, L., and Olin, P. 1998. Levels of anti-pertussis antibodies related to protection after household exposure to Bordetella pertussis. Vaccine. 16:1907-1916.

17. Long, S.S., Welkon, C.J., and Clark, J.L. 1990. Widespread silent transmission of pertussis in families: antibody correlates of infection and symptomatology. J. Infect. Dis. 161:480-486.

18. Leef, M., Elkins, K.L., Barbic, J., and Shahin, R.D. 2000. Protective immunity to Bordetella pertussis requires both B cells and CD4(+) T cells for key functions other than specific antibody production. J. Exp. Med. 191:1841-1852.

19. Mahon, B.P., Sheahan, B.J., Griffin, F., Murphy, G., 
and Mills, K.H. 1997. Atypical disease after Bordetella pertussis respiratory infection of mice with targeted disruptions of interferon-gamma receptor or immunoglobulin mu chain genes. J. Exp. Med. 186:1843-1851.

20. Kirimanjeswara, G.S., Mann, P.B., and Harvill, E.T. 2003. Role of antibodies in immunity to Bordetella infections. Infect. Immun. 71:1719-1724.

21. King, A.J., et al. 2001. Role of the polymorphic region 1 of the Bordetella pertussis protein pertactin in immunity. Microbiology. 147:2885-2895.

22. Musser, J.M., Hewlett, E.L., Peppler, M.S., and Selander, R.K. 1986. Genetic diversity and relationships in populations of Bordetella spp. J. Bacteriol. 166:230-237.

23. van der Zee, A., Mooi, F., Van Embden, J., and Musser, J. 1997. Molecular evolution and host adaptation of Bordetella spp.: phylogenetic analysis using multilocus enzyme electrophoresis and typing with three insertion sequences. J. Bacteriol. 179:6609-6617.

24. Parkhill, J., et al. 2003. Comparative analysis of the genome sequences of Bordetella pertussis, Bordetella parapertussis and Bordetella bronchiseptica. Nat. Genet. 35:32-40.

25. Pishko, E.J., et al. 2004. Antibody-mediated bacterial clearance from the lower respiratory tract of mice requires complement component C3. Eur. J. Immunol. 34:184-193.

26. Kirimanjeswara, G.S., et al. 2005. The complex mechanism of antibody-mediated clearance of Bordetella from the lungs requires TLR4. J. Immunol. In press.

27. Moss, J., et al. 1983. Activation by thiol of the latent NAD glycohydrolase and ADP-ribosyltransferase activities of Bordetella pertussis toxin (islet-activating protein). J. Biol. Chem. 258:11879-11882.

28. Murayama, T., Katada, T., and Ui, M. 1983. Guanine nucleotide activation and inhibition of adenylate cyclase as modified by islet-activating protein, pertussis toxin, in mouse $3 \mathrm{~T} 3$ fibroblasts. Arch. Biochem. Biophys. 221:381-390.

29. Spangrude, G.J., Sacchi, F., Hill, H.R., Van Epps, D.E., and Daynes, R.A. 1985. Inhibition of lymphocyte and neutrophil chemotaxis by pertussis toxin. J. Immunol. 135:4135-4143.

30. Brito, G.A., et al. 1997. Role of pertussis toxin A subunit in neutrophil migration and vascular permeability. Infect. Immun. 65:1114-1118.

31. Meade, B.D., Kind, P.D., and Manclark, C.R. 1984. Lymphocytosis-promoting factor of Bordetella pertussis alters mononuclear phagocyte circulation and response to inflammation. Infect. Immun. 46:733-739.

32. Hewlett, E.L. 1999. A commentary on the pathogenesis of pertussis. Clin. Infect. Dis. 28(Suppl. 2): S94-S98.

33. Cherry, J.D. 1997. Comparative efficacy of acellular pertussis vaccines: an analysis of recent trials. Pediatr. Infect. Dis. J. 16(4 Suppl.):S90-S96.

34. Taranger, J., et al. 2001. Mass vaccination of children with pertussis toxoid--decreased incidence in both vaccinated and nonvaccinated persons. Clin. Infect. Dis. 33:1004-1010.

35. Ravetch, J.V. 1997. Fc receptors. Curr. Opin. Immunol. 9:121-125.

36. Harvill, E.T., Cotter, P.A., and Miller, J.F. 1999. Pregenomic comparative analysis between bordetella bronchiseptica RB50 and Bordetella pertus- sis tohama I in murine models of respiratory tract infection. Infect. Immun. 67:6109-6118.

37. Conlan, J.W. 1997. Critical roles of neutrophils in host defense against experimental systemic infections of mice by Listeria monocytogenes, Salmonella typhimurium, and Yersinia enterocolitica. Infect. Immun. 65:630-635.

38. Seiler, P., et al. 2000. Rapid neutrophil response controls fast-replicating intracellular bacteria but not slow-replicating Mycobacterium tuberculosis. J. Infect. Dis. 181:671-680.

39. Carbonetti, N.H., Artamonova, G.V., Mays, R.M., and Worthington, Z.E. 2003. Pertussis toxin plays an early role in respiratory tract colonization by Bordetella pertussis. Infect. Immun. 71:6358-6366.

40. McGuirk, P., and Mills, K.H. 2000. A regulatory role for interleukin 4 in differential inflammatory responses in the lung following infection of mice primed with Th1- or Th2-inducing pertussis vaccines. Infect. Immun. 68:1383-1390.

41. Belcher, C.E., et al. 2000. The transcriptional responses of respiratory epithelial cells to Bordetella pertussis reveal host defensive and pathogen counter-defensive strategies. Proc. Natl. Acad. Sci. U. S. A. 97:13847-13852.

42. Agarwal, R.K., Sun, S.H., Su, S.B., Chan, C.C., and Caspi, R.R. 2002. Pertussis toxin alters the innate and the adaptive immune responses in a pertussisdependent model of autoimmunity. J. Neuroimmunol. 129:133-140.

43. Shen, W., Bendall, L.J., Gottlieb, D.J., and Bradstock, K.F. 2001. The chemokine receptor CXCR4 enhances integrin-mediated in vitro adhesion and facilitates engraftment of leukemic precursor-B cells in the bone marrow. Exp. Hematol. 29:1439-1447.

44. Pizza, M., et al. 1989. Mutants of pertussis toxin suitable for vaccine development. Science. 246:497-500.

45. Carbonetti, N.H., et al. 2004. Suppression of serum antibody responses by pertussis toxin after respiratory tract colonization by Bordetella pertussis and identification of an immunodominant lipoprotein. Infect. Immun. 72:3350-3358.

46. Nencioni, L., et al. 1990. Characterization of genetically inactivated pertussis toxin mutants: candidates for a new vaccine against whooping cough. Infect. Immun. 58:1308-1315.

47. Ausiello, C.M., et al. 2002. Native and genetically inactivated pertussis toxins induce human dendritic cell maturation and synergize with lipopolysaccharide in promoting $\mathrm{T}$ helper type 1 responses. J. Infect. Dis. 186:351-360.

48. Bokoch, G.M., and Gilman, A.G. 1984. Inhibition of receptor-mediated release of arachidonic acid by pertussis toxin. Cell. 39:301-308.

49. Katada, T., Tamura, M., and Ui, M. 1983. The A protomer of islet-activating protein, pertussis toxin, as an active peptide catalyzing ADP-ribosylation of a membrane protein. Arch. Biochem. Biophys. 224:290-298.

50. Boldrick, J.C., et al. 2002. Stereotyped and specific gene expression programs in human innate immune responses to bacteria. Proc. Natl. Acad. Sci. U. S. A. 99:972-977.

51. Smith, M.L., Olson, T.S., and Ley, K. 2004. CXCR2and E-selectin-induced neutrophil arrest during inflammation in vivo. J. Exp. Med. 200:935-939.

52. Miller, L.A., Usachenko, J., McDonald, R.J., and Hyde, D.M. 2000. Trafficking of neutrophils across airway epithelium is dependent upon both thioredoxin- and pertussis toxin-sensitive signaling mechanisms. J. Leukoc. Biol. 68:201-208.

53. Lawrence, G.L., MacIntyre, C.R., Hull, B.P., and McIntyre, P.B. 2003. Measles vaccination coverage among five-year-old children: implications for disease elimination in Australia. Aust. N. Z. J. Public Health. 27:413-418.

54. Glass, K., and Grenfell, B.T. 2004. Waning immunity and subclinical measles infections in England. Vaccine. 22:4110-4116.

55. Bolker, B.M., and Grenfell, B.T. 1993. Chaos and biological complexity in measles dynamics. Proc. Biol. Sci. 251:75-81.

56. Keeling, M.J., and Grenfell, B.T. 1997. Disease extinction and community size: modeling the persistence of measles. Science. 275:65-67.

57. Bjornstad, O.N., Peltonen, M., Liebhold, A.M., and Baltensweiler, W. 2002. Waves of larch budmoth outbreaks in the European alps. Science. 298:1020-1023.

58. Finkenstadt, B.F., Bjornstad, O.N., and Grenfell, B.T. 2002. A stochastic model for extinction and recurrence of epidemics: estimation and inference for measles outbreaks. Biostatistics. 3:493-510.

59. Storsaeter, J., Hallander, H.O., Gustafsson, L., and Olin, P. 2003. Low levels of antipertussis antibodies plus lack of history of pertussis correlate with susceptibility after household exposure to Bordetella pertussis. Vaccine. 21:3542-3549.

60. Taranger, J., et al. 2001. Immunologic and epidemiologic experience of vaccination with a monocomponent pertussis toxoid vaccine. Pediatrics. 108:E115.

61. Le, T., et al. 2004. Immune responses and antibody decay after immunization of adolescents and adults with an acellular pertussis vaccine: the APERT Study. J. Infect. Dis. 190:535-544.

62. Robbins, J.B., et al. 2005. The diphtheria and pertussis components of diphtheria-tetanus toxoidspertussis vaccine should be genetically inactivated mutant toxins. J. Infect. Dis. 191:81-88.

63. Hausman, S.Z., and Burns, D.L. 2000. Use of pertussis toxin encoded by ptx genes from Bordetella bronchiseptica to model the effects of antigenic drift of pertussis toxin on antibody neutralization. Infect. Immun. 68:3763-3767.

64. Circolo, A., et al. 1999. Genetic disruption of the murine complement $\mathrm{C} 3$ promoter region generates deficient mice with extrahepatic expression of C3 mRNA. Immunopharmacology. 42:135-149.

65. Hodge, L.M., and Simecka, J.W. 2002. Role of upper and lower respiratory tract immunity in resistance to Mycoplasma respiratory disease. J. Infect. Dis. 186:290-294.

66. Mbawuike, I.N., and Herscowitz, H.B. 1989. MH-S, a murine alveolar macrophage cell line: morphological, cytochemical, and functional characteristics. J. Leukoc. Biol. 46:119-127.

67. Rubin-Bejerano, I., Fraser, I., Grisafi, P., and Fink, G.R. 2003. Phagocytosis by neutrophils induces an amino acid deprivation response in Saccharomyces cerevisiae and Candida albicans. Proc. Natl. Acad. Sci. U. S. A. 100:11007-11012.

68. Laichalk, L.L., et al. 1998. Intrapulmonary delivery of tumor necrosis factor agonist peptide augments host defense in murine gram-negative bacterial pneumonia. Infect. Immun. 66:2822-2826. 